\title{
MIGRACIÓN DEL DISPOSITIVO INTRAUTERINO
}

Hernán Araméndiz A. MD*, Hernando Ruiz M. MD**, Pilar Aguilar, MD***

\section{Resumen}

Se plantea el caso de una paciente manejada en el servicio de ginecología del Hospital de San José con diagnóstico de perforación postinserción y migración de DIU. Es una eventualidad poco frecuente y la tasa reportada es de 0.87 por cada 1.000 inserciones. ${ }^{1}$ La perforación postinserción se clasifica como primaria si los síntomas se presentan de manera inmediata o secundaria cuando la migración es consecuencia de contracciones reactivas del útero y la sintomatología se manifiesta de manera tardía. ${ }^{2}$ Para su extracción, según su localización y complicaciones conocidas, se requiere personal calificado y experiencia quirúrgica. A través de la ginecología endoscópica se ha logrado disminuir la extracción a través de laparotomía. Sin embargo, en ciertas ocasiones, como se expone en este caso, no se logra su ubicación o se requiere de otros procedimientos reparativos, según los hallazgos o complicaciones encontrados en la intervención.

Palabras clave: DIU (dispositivo intrauterino), complicaciones, intrabdominal.

\section{Caso clínico}

Se trata de una paciente de 30 años grávida dos, partos dos por cesárea, a quien por deseo de planificación familiar con método no definitivo se insertó DIU en mayo de 2004 sin evidencia clínica de complicaciones. En el control citológico realizado en julio de 2004 no se visualizaron los hilos del dispositivo y se remitió a valoración por ginecología, donde solicitaron ecografía que informó útero en retroversoflexión de $65 \times 41 \times 48 \mathrm{~mm}$, cavidad endometrial vacía, anexos sin alteraciones, sin detectar el DIU en la pelvis. En enero de 2005 la placa de abdomen simple reveló la

Fecha recibido: octubre 6 de 2006 - Fecha aceptado: diciembre I5 de 2006

* Ginecólogo adscrito. Instructor de ginecología endoscópica. Depto. de ginecología y Obstetricia, Hospital de San José.

** Ginecólogo adscrito, coordinador ginecología endoscópica. Hospital de San José.

*** Residente III de Ginecología y Obstetricia. Fundación Universitaria de Ciencias de la Salud, Hospital de San José. presencia del DIU en la pelvis menor. (Figura 1). Se decidió practicar laparoscopia, procedimiento llevado a cabo en abril de 2005, hallando el DIU migrado entre peritoneo de pared anterior y recto sigmoide. Se consultó con cirugía general y se decidió llevar a la paciente, previa preparación de colon, a un segundo tiempo quirúrgico para laparotomía exploratoria. Se programó y se ejecutó la nueva intervención en julio de 2005, sin encontrar el DIU pese a minuciosa y sistémica revisión de la cavidad. Las radiografías tomadas durante la cirugía y el postoperatorio, así como la ecografía abdominopélvica no demostraron el DIU, por lo cual se dio salida por evolución adecuada.

Se considera que el caso clínico expuesto corresponde a DIU con perforación secundaria y migración del útero a la cavidad abdominal, con lesión asintomática del colon sigmoide y posterior ubicación intraluminal y expulsión inadvertida del mismo durante deposición. 


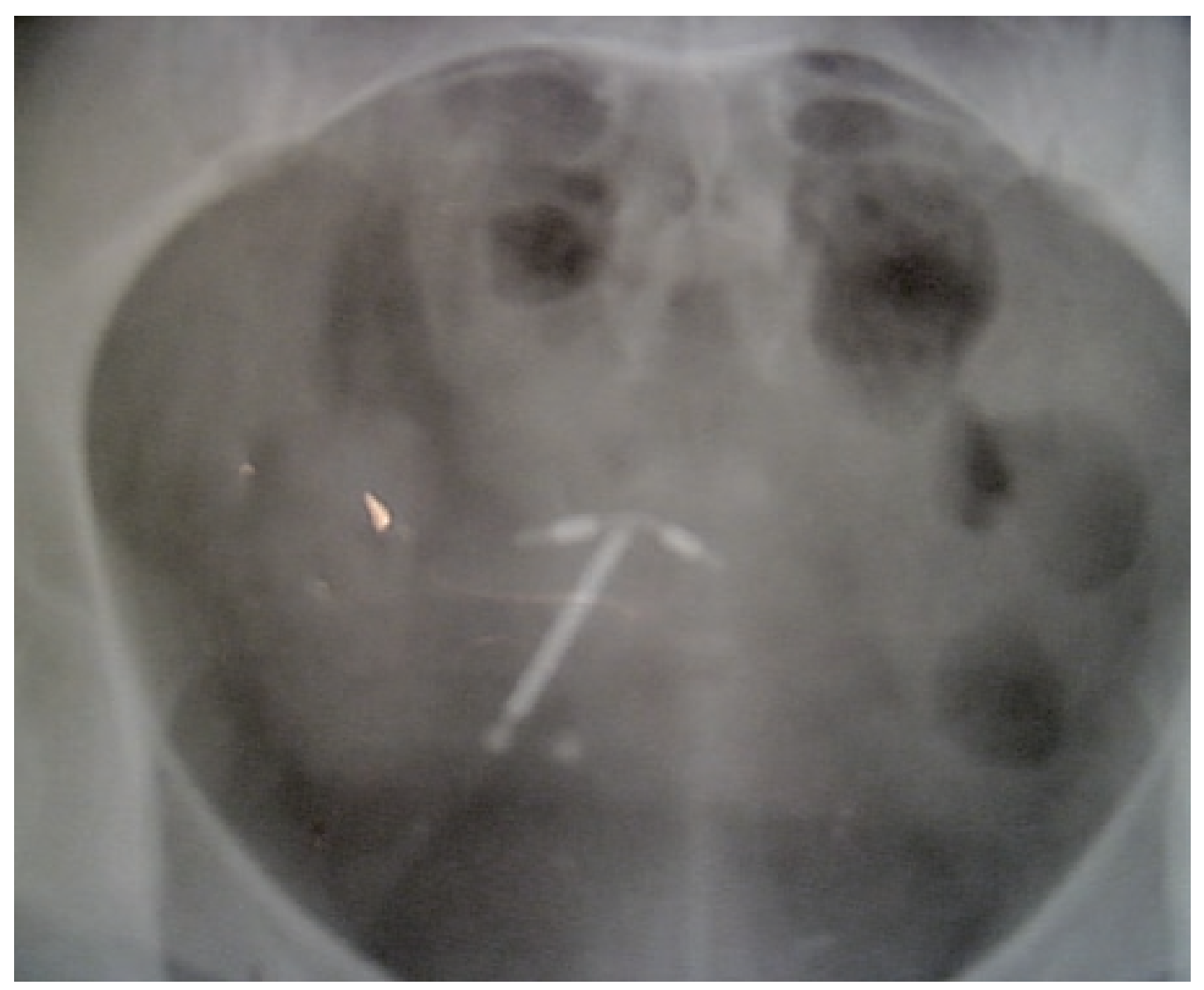

Figura I. Presencia del DIU en pelvis menor.

\section{Discusión}

El DIU es uno de los métodos más comunes de planificación reversible. ${ }^{3}$ El riesgo de perforación depende del tipo de dispositivo empleado, la experiencia del operador que inserta el DIU, la posición del útero y el tiempo de colocación con respecto al momento del parto. ${ }^{4} \mathrm{La}$ incidencia de esta complicación varía de 0.2 a 0.87 por cada 1.000 inserciones, aunque se reconoce la existencia de un subregistro importante. ${ }^{1}$

Se considera primaria si ocurre durante la inserción del DIU por lesión penetrante sobre el tejido miometrial. La presentación clínica consiste en dolor intenso y súbito, asociado con sangrado genital. ${ }^{2}$ En contraste, en la perforación secundaria el intervalo entre la inserción y el diagnóstico de la perforación varía desde días hasta 16 años, como el caso repor- tado de perforación vesical. ${ }^{5}$ El mecanismo de lesión es consecuencia de una reacción a cuerpo extraño por parte del útero y la presión ejercida por el DIU causa abrasión y erosión de la capa endometrial y con una microperforación. La respuesta miometrial causa contracciones uterinas que facilitan la migración hacia la cavidad abdominal. ${ }^{2}$

La mayoría de las publicaciones coinciden en la poca o vaga sintomatología al hacer referencia a la perforación secundaria. La migración puede darse hacia la cavidad peritoneal o a órganos cercanos como el intestino delgado, el apéndice, el colon sigmoide, el recto o a la vejiga, siendo esta última la menos frecuente..$^{5}$ La duración de la migración del DIU es desconocida y puede cursar asintomática por años o manifestarse clínicamente con la sintomatología propia de complicaciones como absceso pélvico, perforación intestinal, fístula vesicouteriuna o como 
se refiere en nuestro caso al ser expulsado de cavidad a través del lumen intestinal.,

En la actualidad la ultrasonografía transvaginal y transabdominal son métodos diagnósticos no invasivos muy confiables para detectar la migración abdominopélvica del DIU, pero el método de elección lo constituye la radiografía simple de abdomen. El estado del arte para el manejo del DIU migrado posiciona a la cirugía mínimamente invasiva como el método ideal para estos casos, pero es responsabilidad del equipo quirúrgico establecer los alcances del procedimiento y no emprender actos heroicos e innecesarios que podrían en muchos casos acarrear complicaciones catastróficas. Se torna entonces en una necesidad evidente que el manejo del DIU migrado se lleve a cabo por un equipo quirúrgico calificado y multidisciplinario, con la capacidad de suspender un procedimiento ante el inminente riesgo de perforación y programar un segundo tiempo quirúrgico por laparotomía con la debida preparación del colon.

Para reducir la incidencia de complicaciones asociadas con el DIU, se requiere adecuado entrenamiento en técnica de inserción, conocimiento del caso clínico, una juiciosa valoración física de la paciente y seguimiento ultrasonográfico. Por último, es importante la explicación oportuna con indicaciones claras y signos de alarma para la paciente.

\section{Referencias}

1. Markovitch O, Klein Z, Gidoni Y, Holzinger M, Beyth Y. Extrauterine mislocated IUD: is surgical removal mandatory? Contraception. 2002 Aug;66(2):105-8.

2. Grimaldi, MD, et al. Unusual Uterine perforation with Multiload $\mathrm{Cu}$ 375R. Am J Forensi Med Pathol, volume 26 (4). December 2005, pp 365-366.

3. Grimaldi L, De Giorgio F, Andreotta P, D'Alessio MC, Piscicelli C, Pascali VL. Medicolegal aspects of an unusual uterine perforation with multiload-Cu 375R. Am J Forensic Med Pathol. 2005 Dec;26(4):365-6.

4. Sogaard K. Unrecognized perforation of the uterine and rectal walls by an intrauterine contraceptive device. Acta Obstet Gynecol Scand. 1993 Jan;72(1):55-6.

5. Harrison-Woolrych M, Ashton J, Coulter D Uterine perforation on intrauterine device insertion: is the incidence higher than previously reported? Contraception. 2003 Jan;67(1):53-6.

6. Mahmutyazicioglu K, Ozdemir H, Ozkan P. Migration of an intrauterine contraceptive device to the urinary bladder: sonographic findings. J Clin Ultrasound. 2002 Oct;30(8):496-8.

7. Ohana E, Sheiner E, Leron E,Mazor M. Appedinx perforation by an intrauterine contraceptive device. Eur J Obstet Gynecol Reprod Biol. 2000 Feb;88(2):129-31.
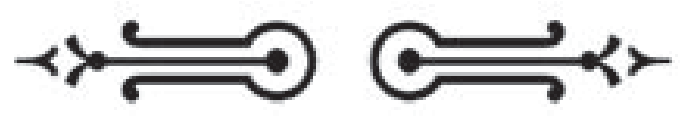